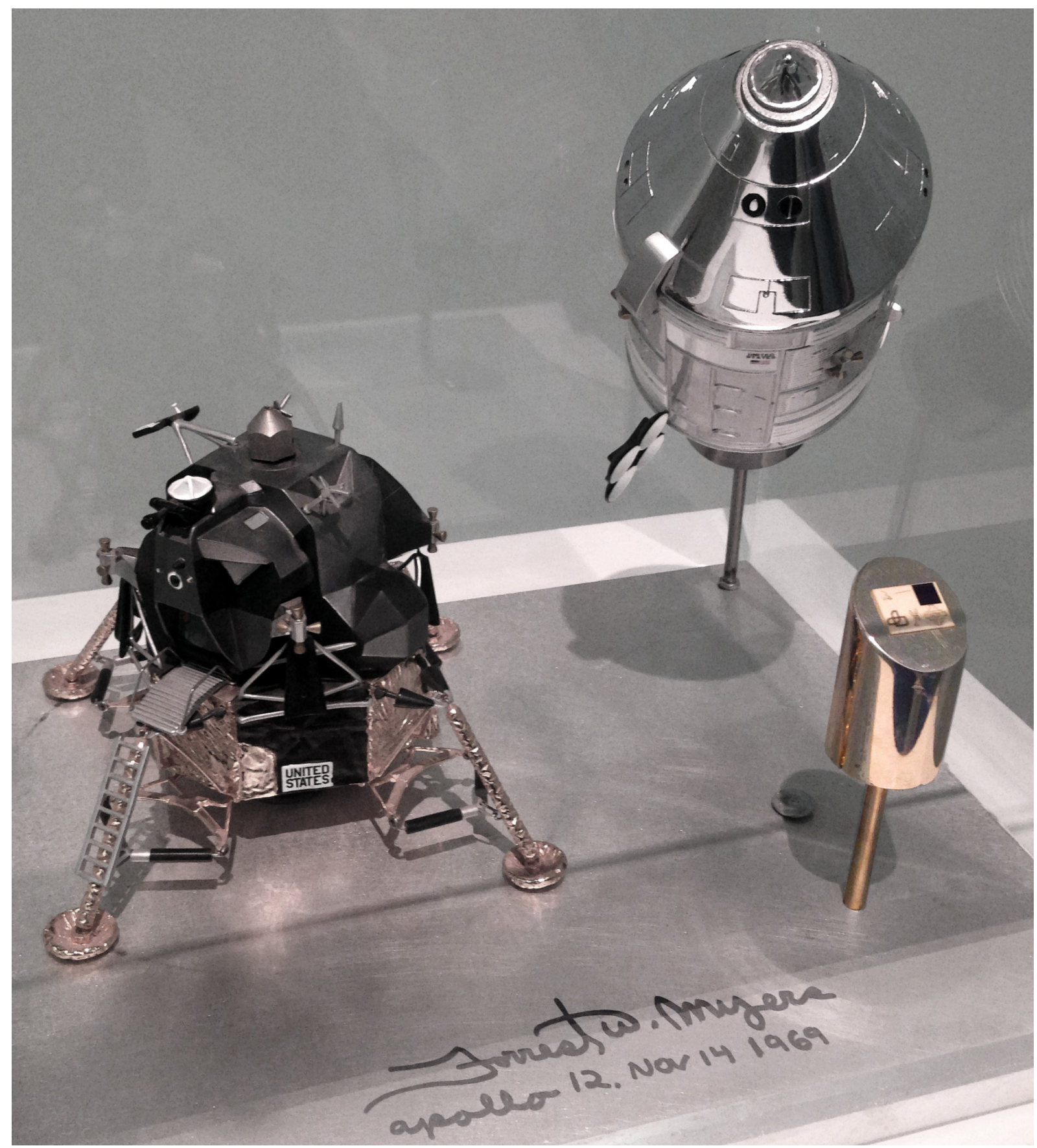




\section{¿Un museo en la luna? The Moon Museum, espacio expositivo en el límite Rafael Guridi}

La idea de un museo en la Luna parece sacada de una película o novela barata de ciencia-ficción, y sin embargo, lo sorprendente es que es una realidad: la historia de una colección de obras de seis de los mas importantes artistas americanos de la década, miniaturizadas en una placa cerámica del tamaño de un chip y colocada subrepticiamente en una de las patas del módulo lunar de la misión Apolo 12, en noviembre de 1969 (segunda misión tripulada a la Luna). Su tamaño, instalación y características, que incluyen la ¿exposición? de obras de seis autores de innegable nombradía llevan al cuestionamiento de la noción misma de museo que su nombre enuncia, a la vez que constituyen una de las aventuras más apasionantes de la Historia del Arte del siglo veinte, encarnado el espíritu de una década de optimismo y fe en la capacidad redentora de la tecnología.

\section{PALABRAS CLAVE}

Museo, Luna, espacio, tecnología, años sesenta

KEYWORDS

Museum, Moon, Space, Tecnologie, Sixties

\begin{abstract}
"The Moon Museum.1969. Various Artists with Andy Warhol, Claes Oldenburg, David Novros, Forrest Myers, Robert Rauschenberg, John Chamberlain. The Moon Museum is thought to be the first artwork to have traveled to the moon. American sculptor Forrest Myers worked with scientists from Bell Laboratories to produce an edition of tiny ceramic tiles onto which drawings by him and five other artists were inscribed. He reported that he had one of the tiles covertly attached to the Apollo 12 spacecraft and that it was left on the moon along with other personal effects transported by the astronauts".
\end{abstract}

MoMA Collection Files

El 19 de noviembre de 1962, el módulo lunar "Intrepid" de la misión Apolo 12 se posaba sobre la superficie lunar. Tres días más tarde, durante su viaje de regreso a la tierra, el periódico New York Times informaba de que habría transportado ocultamente el primer "museo de la luna", un chip cerámico con reproducciones miniaturizadas de Andy Warhol, Claes Oldenburg, David Novros, Robert Rauschenberg, John

\section{Rafael Guridi García}

Arquitecto y Dr. Arquitecto por la ETSAM, Tesis calificada sobresaliente cum laude, menciones de honor en Premio Tesis doctorales UPM y Premio Nacional Bienal Arquitesis, Fundación Arquia. Profesor de Proyectos Arquitectónicos (ETSAM desde 1998); Profesor de Teoría y Técnicas de intervención (ETSAM 2005 a 2010), profesor de Fundamentos de Intervencion (ETSAM, desde 2010); profesor en el Master de Proyectos Arquitectónicos Avanzados y Trabajo Fin de Grado. Miembro del grupo de investigación "Teoría y Crítica de la Arquitectura", miembro de la Cátedra Universidad-Empresa- G+I_PAI sobre patrimonio industrial, miembro de la Red Iberoamericana de Patrimonio PHI, Vocal en el Plan Nacional de Patrimonio Industrial. Profesor invitado en universidades internacionales: Universidad Técnica de Múnich (marzo, 2012), Universidad del Litoral, Argentina (junio, 2013), Universidad de Tongji, Shanghai, (noviembe 2013 a enero de 2014); visiting Scholar en UC Berkeley (becado por la UPM) (2017-18). Orcid ID 0000-0001-6033-3948

Fig. 01

Maqueta de Modulo Intrepid de Apolo 12, y placa del Moon Museum, sobre firma de Forrest Myers, Exposición The Moon Museum en: E.A.T. Experiments in Art and Technology, Museum der Moderne Salzburg. 
Chamberlain y Forrest Myers. La historia de su realización y las maniobras para su instalación tiene todos los ingredientes de una película de misterio, siendo uno de ellos la pregunta de si el Moon Museum ha sobrevivido las duras condiciones del viaje y permanece allí (nadie ha podido viajar para comprobarlo), a pesar de que está acreditada la operación para su instalación, como atestiguan instituciones y medios de prestigio como el propio New York Times o el MoMA de NY (fig. 01). Su existencia rompería varios records: el museo más alejado de cualquier punto del mundo, el menos visitado o el más diminuto de, al menos, el sistema solar. Su tamaño y características llevarían la definición misma de museo, que su nombre enuncia, al límite, a la vez que la ambición que lo anima encarnaría el espíritu de toda una década.

HISTORIA

La idea parte del escultor Forrest "Frosty" Meyers, natural de Long Beach (California, 1941), formado en el San Francisco Art Institute, pero residente en Nueva York desde el principio de la década de los sesenta. En 1969, Meyers participa en los encuentros E.A.T., Experiments in Art

and Technology, organizados por Johan Wilhelm "Billy" Kluver, un ingeniero de Bell Telephone Laboratories, donde se buscaba una integración de Arte y Tecnología por medio de cooperación de artistas e ingenieros en proyectos creativos contemporáneos ${ }^{2}$. Según menciona en sus memorias ${ }^{3}$, Myers estaba obsesionado con los viajes espaciales desde las misiones soviéticas Sputnik de la década anterior, de manera que propone, en el mismo año de la llegada del hombre a la luna, la instalación de un diminuto museo en el satélite, transportada por la siguiente misión espacial programada, Apolo 12 -de lanzamiento previsto para finales de ese mismo año- y conteniendo obras de algunos de los más importantes artistas americanos del momento. Para ello, habla con Andy Warhol, Claes Oldenburg, David Novros, Robert Rauschenberg y John Chamberlain, que aceptan encantados formar parte de la que será la primera muestra artística lunar.

Myers contacta con la NASA, primero directamente, y luego, por medio de una importante institución, el Metropolitan Museum of Art de Nueva York, cuyo director de la sección de Arte Americano, Henry Geldzahler, defiende la iniciativa. Al acercarse la fecha de lanzamiento sin respuesta, Myers y el grupo de artistas deciden hacerlo ilegalmente: contactan con un ingeniero que trabaja en Grumman Aircraft Corporation, la empresa que fabrica los módulos lunares, cuyo nombre se mantuvo -y aún se mantieneen secreto, citado por su nombre en clave, "John F". (un probable guiño al presidente John Fitzgerald Kennedy, impulsor de las misiones Apolo)4.

Dado el carácter secretivo - por no decir claramente ilegal- de la misión, se resuelve fabricar una mínima pieza con reproducciones de obras de los artistas -incluyendo al propio Myers, para poder ser instalada discretamente en una de las patas del módulo "Intrepid" $\sin$ ser detectada. Para ello, cada uno aporta un dibujo, que es reproducido y miniaturizado en los laboratorios de Bell Telephone. Bajo la dirección de Fred Waldhauer, director del laboratorio, los ingenieros Robert Merkle y Burt Unger logran la impresión en una pieza cerámica de tres cuartos por media pulgada $(19,05 \times 12,7 \mathrm{~mm})$ con un espesor de un cuarentavo de pulgada $(0,64 \mathrm{~mm})$, el tamaño aproximado de una tarjeta SIM de un teléfono móvil (fig. 02). 


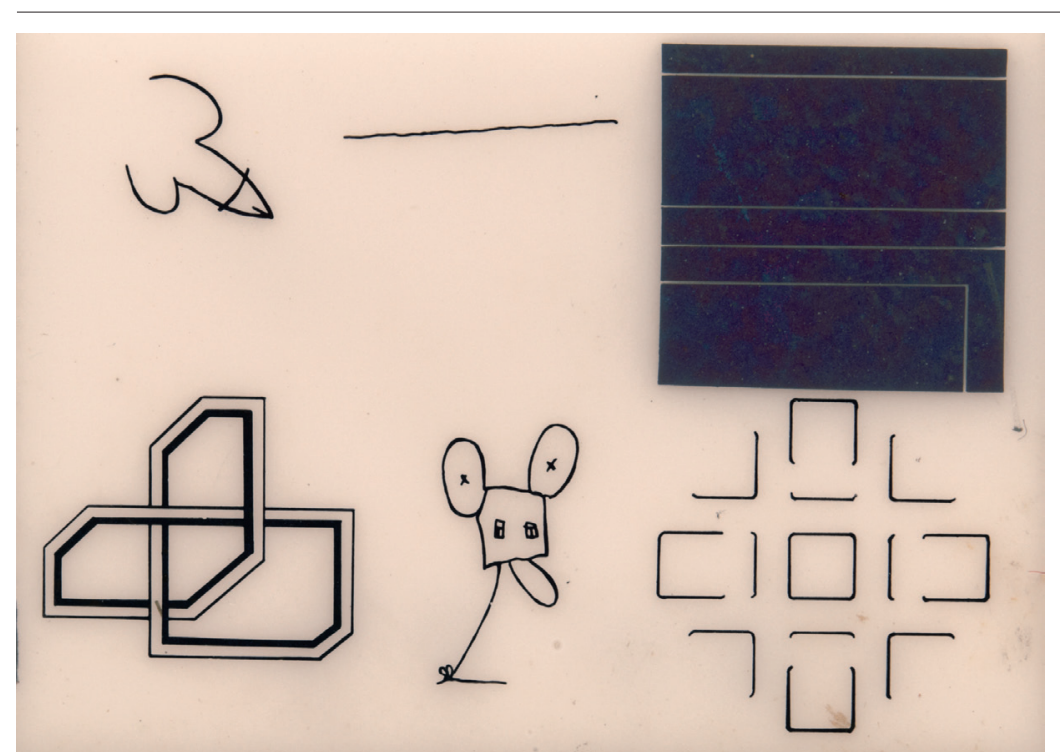

02

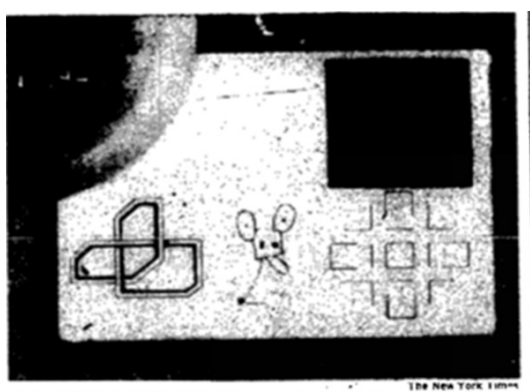

ART ON THE MOON? Clockwise from top center, these designs are a spur-of-the-moment line by Robert Rauschenberg, a square by David Novros, a mouse by Claes Oldenburg, computer drawing by Forrest Myers. Thumb $\alpha$ person holding wafer covers signature of Andy Warhol. According to Mr. Myers, drawings are on leg of Apollo 12 lander.

New York Sculptor Says Intrepid Put Arton Moon BY GRACE GLUECK

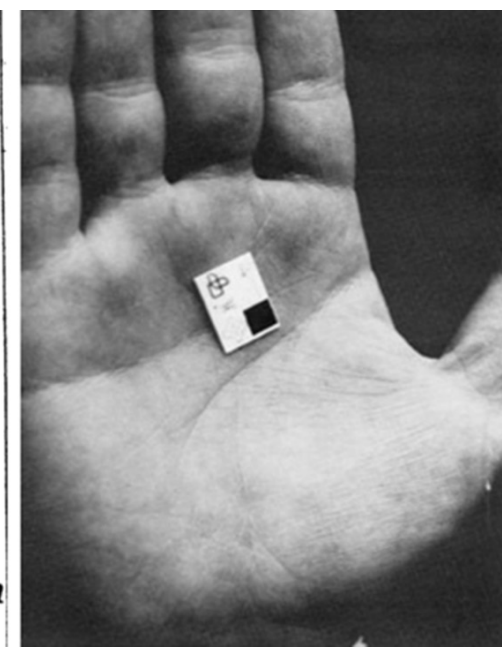

04

Se utilizan técnicas pioneras para circuitos impresos, en los que Robert Merkle era un especialista. Se reducen fotográficamente cada uno de los dibujos para que los seis entren en una pieza rectangular de las dimensiones de la placa, que se montan en unas piezas de vidrio (repitiendo el proceso doce veces). Se preparan otras tantas placas cerámicas de igual dimensión, con una capa metálica de nitruro de tantalio y una capa de material fotosensible. Se montan ambas piezas (vidrio y cerámica) y se exponen a la luz, actuando como una suerte de copia de contacto fotográfico, quedando grabado el dibujo sobre la pieza cerámica de una manera resistente a las extremas condiciones atmosféricas del viaje y futuro emplazamiento ${ }^{5}$.

Se hacen varias copias, y se destruyen los moldes de vidrio a fin de prevenir futuras copias no controladas. Hay discusión sobre el número final de placas, ya que parte de ellas fueron a parar a los técnicos del laboratorio de Bell Telephone. Robert Merkle dice que se hicieron 12 en total ${ }^{6}$, pero Jade Dellinger, director de la Bob Rauschemberg Gallery

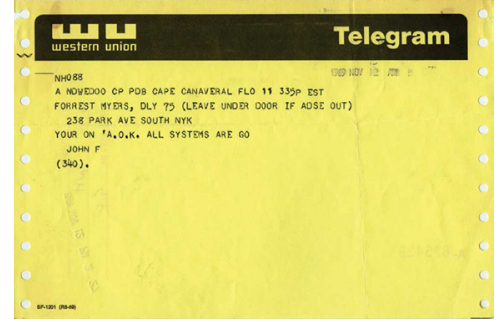

03

Fig. 02

The Moon Museum, archivo MoMA, NY 1969 (Code: 0156592).

Fig. 03

Telegrama de “"'John F”. a Forrest Myers (12/noviembre/1969) comunicandole la colocación del "museo" en el módulo (Wikimedia commons/ Wikipedia).

Fig. 04

The New York Times, fragmento de la publicación de The Moon Museum, 22 de noviembre de 1969 (archivos The New York Times). 
en Florida ha señalado que el número total pudo alcanzar la cuarentena, aunque reconoce que el número de piezas localizadas es mucho menor?.

Finalmente, "John-F", el contacto secreto de los artistas y los técnicos de Bell es destinado en cabo Cañaveral; el 12 de noviembre de 1969, dos días antes del lanzamiento, "John F" envía a Myers un telegrama de confirmación: "YOUR ON ‘A.O.K. ALL SYSTEMS GO” (fig. 03). El telegrama forma parte de la leyenda, ha sido incluido en exposiciones sobre el museo y su texto, incluyendo sus errores sintácticos, ha dado pié a títulos de publicaciones y conferencias ${ }^{8}$.

La nave de la misión Apolo 12, al mando de los astronautas Charles "Pete" Conrad, Alan L. Bean y Richard Gordon despegó el 14 de noviembre a las 16;22 UTC, y alunizó en el Oceanus Procellarum Selenita el 19 de noviembre, a las 06:54 UTC. Desde entonces, el Moon Museum permanece (¿permanecería?) anclado a una de las patas del módulo lunar "Intrepid" sin que, hasta la fecha, nadie haya podido comprobarlo.

Durante el viaje de regreso de la misión, Forrest Myers envía un resumen de los hechos, junto con una reproducción de una de las placas, al diario New York Times, el cual, el 22 de noviembre, publica la noticia: "New York Sculptor Says Intrepid Put Art on Moon", e incluye, con la información, la foto de la placa, dando credibilidad a la historia y origen a la leyenda del primer museo en la Luna9 (fig. 04).

LAS OBRAS

Según Myers, todos los artistas se tomaron muy en serio el proyecto ${ }^{10}$. Debido al diminuto tamaño del soporte y a la técnica de reproducción empleada, todos apostaron por dibujos a línea, muy elementales y en general vinculados a sus intereses personales (fig. 05).

La esquina superior izquierda la encabeza un dibujo de Andy Warhol, en la que conjuga sus iniciales A-W con la forma de un aparato genital masculino. Hay quien ha querido ver simultáneamente la forma elemental de un cohete, lo que le conferiría una mayor coherencia con el proyecto". Siguiendo el sentido de las agujas del reloj, Robert Rauschenberg dibuja una sencilla línea recta horizontal. Para Jade Dellinger, los trabajos de Warhol y Rauschenberg proponen legar una especie de huella conceptual equivalente a las dejadas por las botas de Neil Amstrong en la superficie del satélite ${ }^{12}$.

El resto de artistas presenta dibujos en línea con los trabajos que estaban desarrollando en ese momento. Davis Novros propone una composición en negativo de líneas blancas sobre un rectángulo negro. Para Dellinger, las líneas blancas serían la traducción plana de los espacios que separan las piezas de gran escala de este artista. John Chamberlain presenta un esquema geométrico con ecos de un circuito impreso.

Especialmente relevante es el dibujo de Claes Oldenburg, cuya obra se caracteriza por la ampliación a una escala desmesurada de objetos banales de la cultura popular. Obligado esta vez a trabajar a una escala diminuta, presenta una versión elemental de su gigantesca cabeza estilizada de Mickey Mouse, obra denominada Mouse Museum/ Ray Gun Wing (1965-1977).

Finalmente, el propio Forrest Myers propone un dibujo que presenta una geometría tridimensional, dentro de la serie denominada Computer Drawings. Junto a la de Chamberlain, es la obra más cercana al espíritu tecnológico de la misión. 


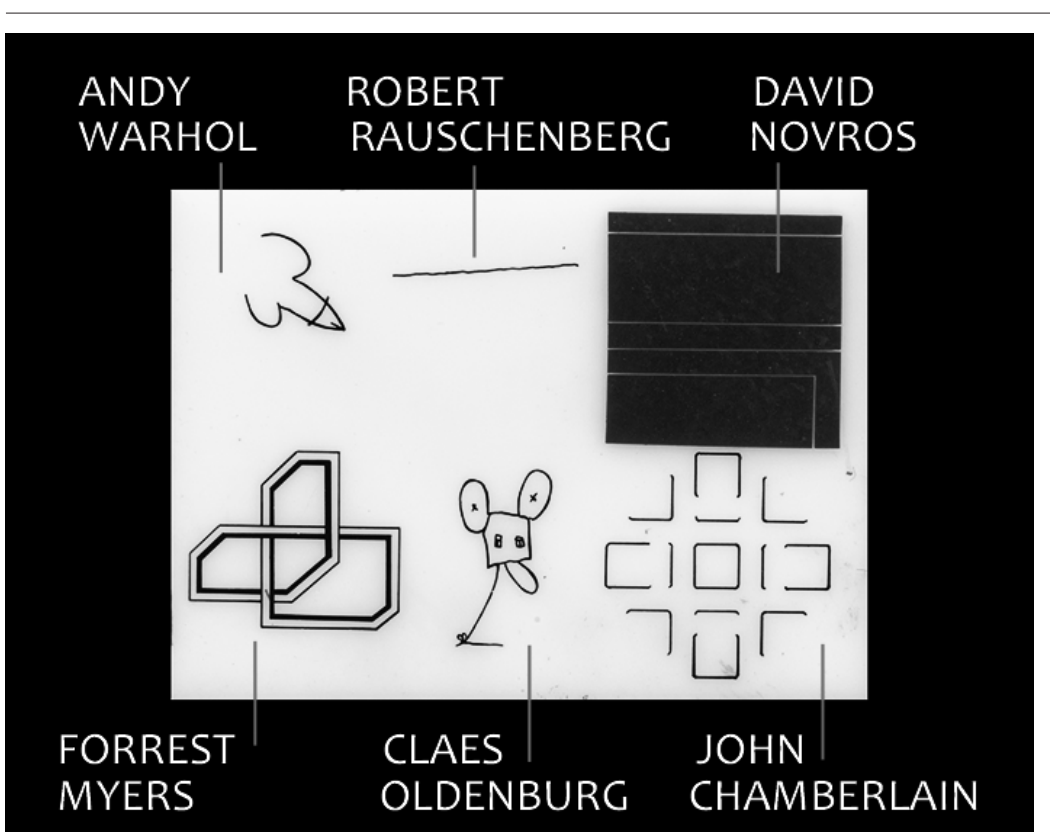

05

Hay que señalar que, en la reproducción de la pieza de la publicación del New York Times, un dedo pulgar sujeta el chip por su esquina superior izquierda, ocultando precisamente el dibujo fálico de Andy Warhol.

¿MUSEO SIN VISITANTES?
La idea de un museo sin visitantes parece, de entrada, una contradicción. Los más importantes diccionarios definen la entrada "Museo" siempre vinculada a la exposición pública. El Merrian Webster, referencia en lengua inglesa, da dos acepciones, la segunda de las cuales

define "Museum" como "a place were objects are exhibited". El diccionario de la RAE proporciona una definición casi idéntica: "Lugar donde se exhiben objetos o curiosidades" (las otras acepciones refieren a la institución o al edificio).

La idea de museo parecería pues consustancial a la idea de exhibición pública de obras, tanto como la visualización en una obra plástica, la audición en una obra musical o la lectura (o escucha) en una obra literaria. Sin embargo, el siglo XX ha proporcionado numerosos ejemplos que contradicen la anterior afirmación: en 1961, el artista conceptual italiano Piero Manzoni saco al mercado del arte su célebre merda d'artista envasada herméticamente en noventa latas de seis centímetros de diámetro y cinco de alto, al mismo precio (al peso) que el oro, cifras que se han multiplicado en las pocas ocasiones posteriores que han salido al mercado. Al parecer ninguna lata ha sido abierta para ver (y verificar) su contenido. La también célebre obra musical 4'33 " de John Cage (1952) consta de cuatro minutos, treinta y tres segundos de absoluto silencio, repartidos en tres movimientos. Dado que la música es una combinación de sonidos y silencios pautados por distintos tiempos, nada impide una obra con un único recurso a estos últimos. La obra Bartleby y Compañía, de Enrique Vilamatas (2001), recoge una selección de escrito- 


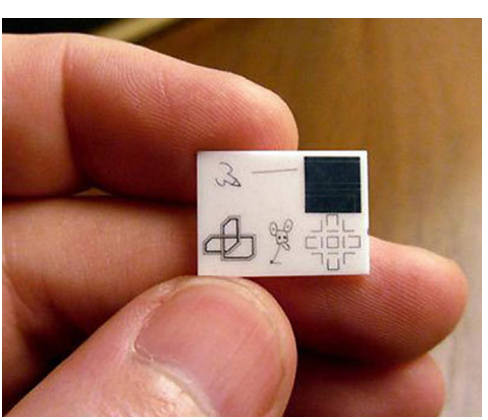

06

Fig. 06

Moon art scale fingers, http://www. forevergeek.com/2010/06/nasa_ astronauts_smuggled_artwork_onto_the_ moon/ res ágrafos, si ello es posible. Aparte del prólogo, el libro solo es legible en las notas a pié de página que acompañan un texto, coherentemente, en blanco. En 2003, el artista Santiago Sierra fue nombrado comisario del pabellón de España en la $50^{a}$ edición de la Bienal de Venecia. El artista decidió no realizar montaje expositivo alguno, dejando en el pabellón los restos desnudos del desmontaje de las últimas exposiciones; su actuación se redujo al establecimiento de un riguroso control de entrada a esta "nada" expuesta, de manera que solo se podía acceder al interior de la misma con DNl o pasaporte español.

Obras de arte plástico que no se ven, músicas que no se oyen, escritos sin palabras, exposiciones sin contenido alguno parecerían contradecir la voluntad comunicativa inherente a toda creación artística. Los ejemplos arriba citados ponen de manifiesto una estrategia frecuente en el arte contemporáneo, tendente al desplazamiento de la visibilidad física o explícita y su sustitución por la visibilidad conceptual. La obra de Manzoni cuestiona, no solo los límites de la mercantilización del arte -como el mismo autor señalaba- sino la esencia misma de la artisticidad de un objeto banal, en una línea ya enunciada en 1917 por Marcel Duchamp y su fuente. Sierra señalaba el carácter arbitrario del concepto de frontera, y las kafkianas consecuencias de esta arbitrariedad, mientras que Cage señalaba que 4'33 " enfatizaba el valor del silencio como elemento musical, al tiempo que expresaba su temor de que la obra fuera interpretada como una mera "boutade", de no ser tomado en serio; esa misma seriedad fue la actitud con la que participaron los artistas del Moon Museum, según señalaba Myers (fig. 06).

MUSEO DE AUTOR

La idea del Moon Museum se encuadraría en una línea de trabajo artístico a la que recientemente se ha referido como museos de autor, colecciones particulares de obras propias $y$ ajenas en formatos mínimos, a menudo transportables que operan fuera de los cauces

institucionales, pero que reunirían, al menos en su condición límite, las características mínimas para ser considerados como museos.

La idea fundacional vendría dada de nuevo por Marcel Duchamp y su conocida Boîte-en-Valise (1935-1945) donde el autor reúne una muestra miniaturizada de toda su obra, idea tomada tres décadas después por diversos autores: en 1968, otro Marcel, en este caso Broodthaers inaugura su Musée d'Art Moderne, situado en su casa de Bruselas, que ya incluía piezas ajenas, a menudo objets trouvés o piezas banales.

Más ambicioso y personal, el Schubladenmuseum (museo de los cajones) realizado por el artista suizo Herbert Distel en los años sesenta y setenta propone una selección de piezas de arte contemporáneo de las dos décadas convenientemente miniaturizadas y alojadas en pequeñas cajas cúbicas de unos cinco centímetros de lado, insertadas en veinte cajones que forman un mueble compacto, reuniendo un total de 500 obras $^{13}$.

Muy vinculado al Moon Museum por autoría y concepto, el americano Claes Oldenburg también propone su museo de autor, el Mouse Museum /Ray Gun Wing, concebido entre 1965 y 1977, un contenedor visitable que perfila un estilizado Mickey Mouse, alojando 400 objetos que incluyen desde bocetos y obras del artista hasta readymades y objetos ajenos. Precisamente, es este perfil miniaturizado con 


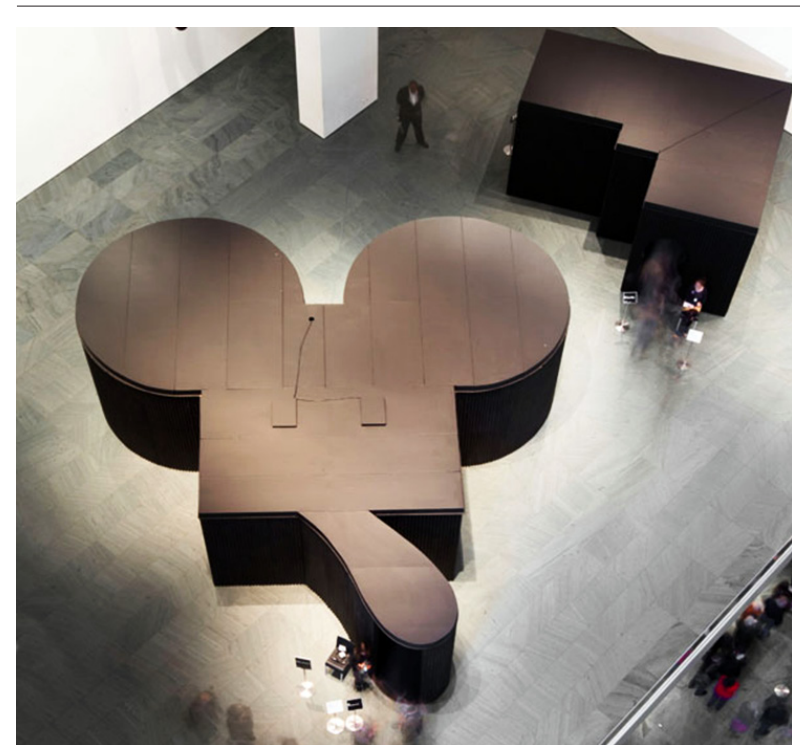

07

un drástico cambio de escala el que el artista aporta para su inclusión en el Moon Museum (fig. 07).

Desde los años sesenta, los museos de autor han proliferado, con ejemplos como el Museo Salinas, dedicado a vituperar la figura del presidente mejicano Salinas de Gortari e inaugurado por el artista también mejicano Vicente Razo en el cuarto de baño de su casa; el artista se auto-nombró director del Museo Salinas y hasta publicó una guía del mismo, the official Museo salinas Guide; como museos personales pueden citarse también el HOMU o Homeless Museum of Art (un carrito portable en plena calle) de Filip Noterdaeme (2002), el Museo de la calle, del colectivo Cambalache (Bogotá, 1999) o el Food Culture Museum, del catalán Antoni Miralda (Expo Hannover, 2000).

Los museos de autor han sido objeto de recientes estudios, como el del mejicano Tomás Ruiz-Rivas ${ }^{14}$, o de exposiciones como la que, bajo el título de Museum Show, celebró el Museo Arnolfini, de septiembre a noviembre de 2011 en la ciudad de Bristol' ${ }^{15}$. Partiendo de la Boîte-en valise, la exposición incluía una buena muestra de mini-museos de autor, que, por supuesto, incluía el Moon Museum.

\section{BACKGROUND}

El programa Apolo viene a culminar y simbolizar una década, la de los años sesenta, caracterizada por un gran optimismo, fe en el progreso científico y gran despegue económico, una vez superados los efectos de la posguerra. Es la era del Baby Boom, un gran crecimiento demográfico y un empoderamiento de las más jóvenes generaciones, algo sin precedentes en la historia reciente; es la década de la revolución pacífica, de la contracultura, el movimiento Beatnik y el Hippismo, la revolución sexual y la relajación de costumbres -conocido en el mundo sajón como los "Swinging Sixties"- los años de la eclosión musical del pop-rock y la psicodelia, de James Bond y el Flower power.

La visión optimista y redentora de la ciencia es un tema trasversal que atraviesa todos los segmentos culturales, desde los más
Fig. 07

Claes Oldenburg: Mouse Museum/Ray Gun Wing at The Museum of Modern Art, New York (April 14-August 5, 2013). Photo: Jason Mandella. @ 2013 The Museum of Modern Art. 


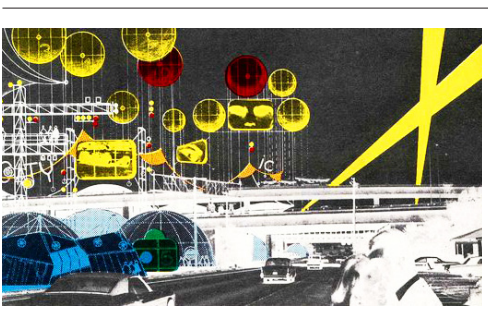

08

Fig. 08

Instant City, Johana Mayer, Archigram, 1955-1968. populares hasta los círculos académicos más selectos. En arquitectura, son los años de Archigram, Superstudio o Archizoom, de Yona Friedman, Frei Otto y Buckminster Fuller, de Kenzo Tange y los metabolistas japoneses Una mirada a la producción de los años finales de la década nos da un perfecto retrato-robot de la época: Expo de Montreal en 1967 (con pabellones de Buckminster Fuller y Frei Otto), y para la que Peter Cook había incluso proyectado una torre. En 1968 James Stirling finaliza la maquinista Biblioteca de Cambridge, Johana Mayer-Archigram presentan su Instant-City, (fig. 08) y Robert Venturi monta en Yale un grupo de investigación, denominado "Learning from Las Vegas or Form Analysis as Design Research". En ese mismo año se extrenan las películas Yellow Submarine (Georges Dunning) y 2001, a Space Odyssey (Stanley Kubrick), que adelantaba una colonización de la Luna descrita con gran precisión científica un año antes de la primera llegada (fig. 09).

El Moon Museum participa del optimismo futurista de sus contemporáneos, constituyendo además una perfecta encarnación de los ideales de los encuentros Arte-Tecnología E.A.T. en los que se gestó la idea. Unido a este ideal, el carácter alegal le confiere un valor añadido, como una acción transgresora o un acto de microterrorismo cultural, inofensivo pero altamente simbólico, que lo enlaza con los valores contestatarios de la época.

El Moon Museum es de los primeros proyectos en vincular arte a la carrera espacial; pronto la idea será seguida por otras iniciativas, aunque con perfiles distintos: en julio de 1971, la misión Apolo 15 pondrá en la luna una nueva pieza artística, Fallen Astronaut, una figurita de aluminio de unos centímetros de altura que representaría un estilizado astronauta. La idea parte de un escultor belga, Paul van Hoeydonck, y su galerista en Nueva York. Al encontrar parecidas trabas a las que se había enfrentado Myers, contactan directamente con los astronautas, los que acceden a depositarlo con ciertas condiciones (mínimo tamaño, ausencia de genero o raza identificable) y se acompañará de una placa con nombres de astronautas soviéticos y americanos muertos en misiones espaciales. El comandante David Scott la depositará en el lugar de aterrizaje -Mare Imbrium-, la fotografiará y lo hará público en la conferencia de prensa celebrada a su regreso. Ignorante del Moon Museum, tanto el comandante Scott como el artista van Hoeydonck reclamarán la colocación del primer objeto artístico en la Luna, a lo que el New York Times responde, reclamando esa condición para el Moon Museum y criticando la acción como una operación de marketing del artista y su galería, bastante desconocida entonces en ambientes artísticos neoyorquinos, polémica acrecentada cuando el escultor pretendió comercializar copias de la estatuilla, a pesar de un pacto previo en contra ${ }^{16}$.

Recientemente, el planeta Marte ha sustituido a la Luna en el objetivo de algunos artistas: en 2003, la Agencia Espacial EuropeaESA lanza la misión Mars Express, con un módulo de aterrizaje, el Beagle 2 , que incorpora una placa de aluminio de unos $10 \times 10$ centímetros con unos círculos de colores (recordando bastante a una paleta de acuarelas) del artista británico Damien Hirst, que debería de servir además para calibrar el equipo de rayos $X$. La señal del Beagle-2 se perdió en el aterrizaje, debido a un probable fallo en la operación.

La placa de Hirst, probablemente aún anclada al módulo ( o a sus restos), tardó poco tiempo en perder su exclusividad en la superficie marciana: en enero de 2004, la NASA lanza un nuevo Rover, denomi- 


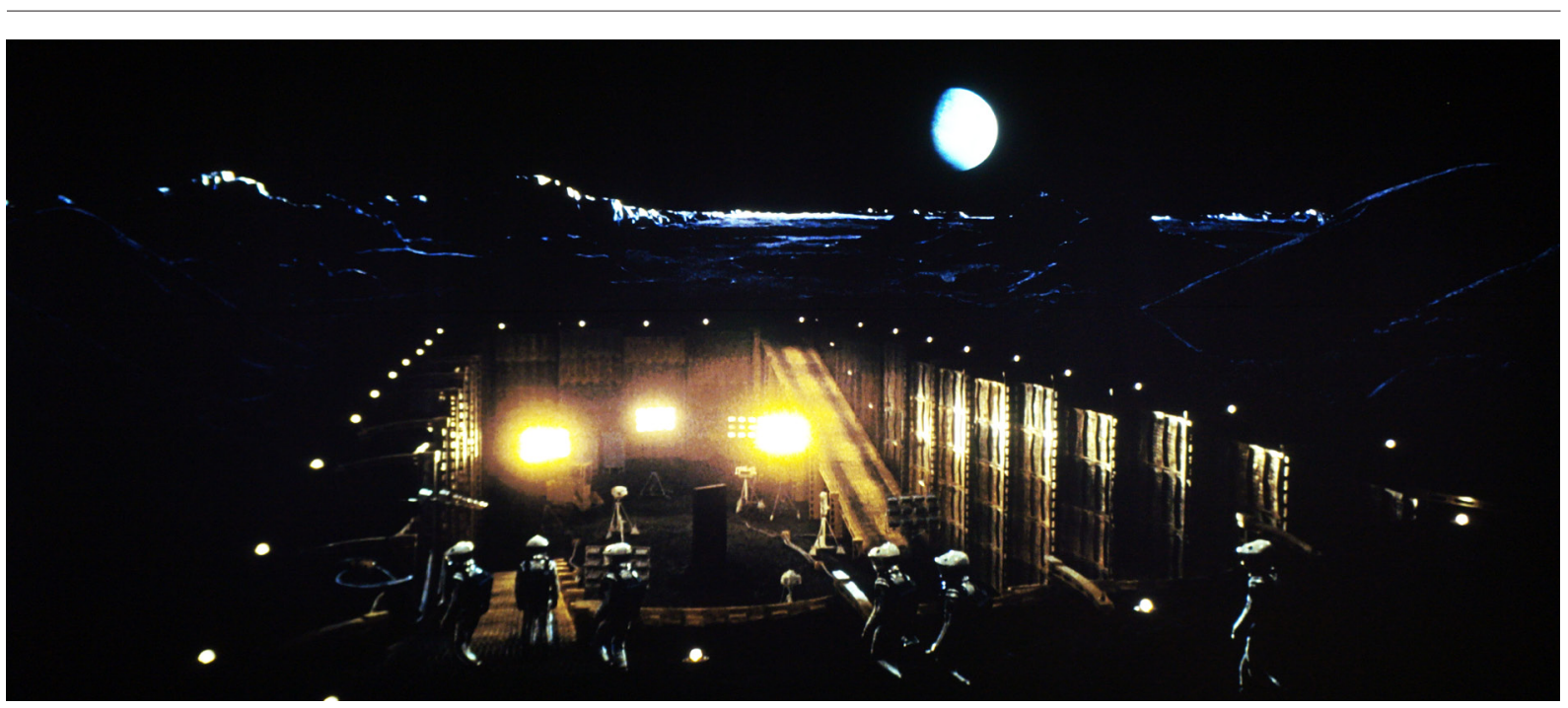

09

nado "Spirit" sobre el planeta rojo, que incorporaba otro objeto artístico; esta vez es un DVD, concebido por el artista australiano (residente en Londres) Stephen Little, conteniendo cuatro millones de nombres de otros tantos voluntarios que respondieron al llamamiento para dejar su nombre en el planeta. El aterrizaje fue esta vez exitoso, y el Rover Spirit ha estado operativo hasta 2011.

Se podría cuestionar el carácter de objeto artístico de ambas creaciones, dado que la placa de Hirst era una herramienta de calibración y el DVD de Little era solo un listado de nombres, pero esa no es la cuestión que aquí nos ocupa.

Habría que recoger aquí un futuro regreso artístico a la Luna de la mano de un mecenas, el millonario japonés Yusaku Maezawa, que planea realizar un viaje privado al satélite acompañado de seis artistas para realizar una acción conjunta. El proyecto Dear Moon no tendrá lugar antes de 2023 y, ni el carácter de la acción ni el nombre de los artistas elegidos han sido desvelados ${ }^{17}$.

Todos estos proyectos mencionados son muy diferentes de las características del Moon Museum: en todos ellos o bien están regidos una iniciativa personal (del artista van Hoeydonc, del promotor Maezawa) cercana a la autopromoción, o bien (como en los casos marcianos), parecería que las dos agencias espaciales, europea o americana, habrían buscado nombres de artistas conocidos en una suerte de técnicas de publicidad: ambos objetos (tabla de color de calibración y registro de nombres) podrían haberse perfectamente llevado a cabo sin la mediación de conocidos artistas.

CONCLUSIONES

En el límite del concepto de "museo", el Moon Museum reúne todas las mínimas características exigibles para ser declarado como tal: una colección de piezas de artistas destacados expuesta como en un museo al ¿aire? libre: la dificultad real de acceso público no invalida su condición museística, ya que un futuro desarrollo tecnológico (condición externa al museo) podría facilitar ese acceso, y de otro lado, existen otros
Fig. 9

2001 una odisea del espacio (Kubrick 1968), escena en la Luna. (Matthew J. Cotter / Flickr2Commons). 
museos de muy difícil visita, como el museo submarino de Lanzarote, o los situados en las casas (y cuarto de baño) de Marcel Broodthaers y Vicente Rizo.

Por otra parte el desplazamiento conceptual sustituye la obra artística por la idea que subyace (el valor del silencio en Cage, la arbitrariedad de la frontera en Sierra). El Moon Museum reclama para si el valor de la tecnología y la aventura científica como parte de su acervo creativo. Lo importante es que el objeto esté en la Luna, que esté puesto en el mismo año de la llegada del hombre al satélite y la manera de lograrlo, en una inteligente operación "parasitaria" que minimiza costes, no hace sino otorgar valor a la aventura conjunta, por encima del valor específico de sus obras individuales. El desplazamiento conceptual permite valorar ante todo la idea del museo, la fe puesta en que el objeto esté realmente allí (una fe parecida, salvando las distancias, al contenido de las latas de Manzoni), al tiempo que - por ser perfectamente reproducible- podemos contemplar la veintena de copias facsímil que circulan por museos y galerías sin necesidad de transportarnos a la Luna.

Diminuto, lejano, de acceso -hoy por hoy-casi imposible, alegal, increíblemente eficiente en su relación coste/ objetivos, el Moon Museum es una de las actuaciones más ambiciosas en el campo artísti$\mathrm{co}$, digno representante de una década de optimismo tecnológico y fe en el futuro que quería llevar la imaginación al poder. RA 


\section{Notas}

01. V.V.A.A., Andy Warhol, Claes Oldenburg, David Novros, Forrest Myers, Robert Rauschenberg, John Chamberlain, The Moon Museum, 1969. The Museum of Modern Art (MoMA); Various Artists, | MoMA". MoMA. https://www.moma.org/ collection/works/62272.

02. KLÜVER, Billy, Experiments in Art and Technology, J. Martin, Barbara Rose (eds.), New York, E. P. Dutton, 1972.

03. MYERS, Forrest. History of the Moon Museum. Forrest Myers Archives. En: http://jiminy.medialab.sciences-po.fr/ eat_datascape/project/295 (última visita: 9 , febrero, 2019).

04. Grumman Aircraft Corporación, fundada en 1929, participó en la construcción de 13 módulos lunares para la NASA, en el programa Apolo. En 1969 cambió su nombre a Grumman Aerospace Corporation, y en 1994, tras una fusión de empresas, paso a llamarse Northrop Grumman.

05. MERKLE, Robert, The History Detectives Team, "Moon Museum Update I History Detectives | PBS “. PBS, 23 juin 2011. http://www.pbs.org/opb/historydetectives/ blog/moon-museum-update/.

06. Ibid.

07. "The total number of copies remains uncertain: as many as forty seem to have been produced, but reports are contradictory and far fewer have actually been located". En: DELLINGER, Jade, et ANNICK Bureaud. "All Systems Are Go: A Museum for the Moon". Festival @ rt outsiders 2009. (In)habitable L'art des environnements extrêmes, 2009. pp. 20-22.

08. DELLINGER, Jade, ANNICK Bureaud, op. cit.

09. GLUECK, Grace, "New York Sculptor Says Intrepid Put Art on Moon", The New York times, November 22 $2^{\text {nd }}, 1969$. En: archivo histórico del periódico: https://www. nytimes.com/1969/11/22/archives/newyork-sculptor-says-intrepid-put-art-onmoon.html?sq=art+on+the+moon\%253F\& $\mathrm{scp}=1 \& \mathrm{st}=\mathrm{p}$ (última visita: 9 , febrero, 2019).

10. DELLINGER, Jade, ANNICK Bureaud, op. cit.

\section{Referencias bibliográficas}

11. "He created a stylized version of his initials which, when viewed at certain angles, can appear as a rocket ship or a penis". STINSON, Elizabeth. "We Sent a Dick Pic to the Moon-And We're Doing It Again" en; WIRED: https://www.wired. com/2015/05/we-sent-a-dick-pic-to-themoon/

12. "Like Neil Armstrong's boot impressions on the lunar surface, both can be interpreted as the conceptual footprint of an artist". DELLINGER, Jade, et ANNICK Bureaud, op. cit.

13. En la actualidad, en el Kunsthaus de Zurich: http://www.kunsthaus.ch/de/ sammlung/restaurierung/beispiele-ausder-praxis/herbert-distel/ (ultima consulta: 10, febrero, 2019).

14. RUIZ RIVAS, Tomas, Museos de artistas, Edición digital del Antimuseo, Ciudad de México 2014. www.antimuseo.org.

15. https://www.arnolfini.org.uk/whatson/ museum-show-part-1 (última consulta: 10, febrero, 2019).

16. Associated Press (21 July 1972). "Commercialism Taints Another Apollo Memento". Modesto Bee. p. 7: en: https://en.wikipedia.org/wiki/Fallen_ Astronaut.

17. https://dearmoon.earth/
- MERKLE, Robert, The History Detectives Team. "Moon Museum Update I History Detectives | PBS “. PBS, 23 June 2011. http:// ww.pbs.org/opb/historydetectives/blog/ moon-museum-update/

- The Museum of Modern Art (MoMA); The Moon Museum, 1969/Various Artists, Andy Warhol, Claes Oldenburg, David Novros, Forrest Myers, Robert Rauschenberg, John Chamberlain. I MoMA. https://www.moma. org/collection/works/62272.

- The New York times, November 22nd, 1969 (archivo histórico del periódico): https://www.nytimes.com/1969/11/22/ archives/new-york-sculptor-says-intrepidput-art-on-moon.html?sq=art+on+the $+m$ oon $\% 253 F \& s c p=1 \& s t=p$.

- Tampa Museum of Art, The Moon Museum: First Space Art Object Lands at Tampa Museum of Art.
RA. Revista de Arquitectura Núm. 21 - 2019 P. $108-119$ 